\title{
Extension of the Deep-Hole Drilling Method for the Measurement of in-Plane Residual Stresses in Composite Laminates
}

\author{
Carlos Garza ${ }^{1, a^{*}}$, Raj Das ${ }^{2, b}$, Martyn J. Pavier ${ }^{1, c}$, Anton Shterenlikht $^{1, \mathrm{~d}}$ and \\ David J. Smith ${ }^{1}(\dagger)$ \\ ${ }^{1}$ Department of Mechanical Engineering, University of Bristol, Queen's Building, University \\ Walk, Bristol, BS8 1TR, United Kingdom \\ ${ }^{2}$ Department of Mechanical Engineering, University of Auckland, Auckland 1010, New Zealand \\ acarlos.garza@bristol.ac.uk, rr.das@auckland.ac.nz, 'martyn.pavier@bristol.ac.uk,
}

(†) Deceased on November $13^{\text {th }}, 2015$

\begin{abstract}
Keywords: Composite Laminates, Deep Hole Drilling, Finite Element Analysis, Residual Stress
\end{abstract}

\begin{abstract}
Deep-Hole Drilling (DHD) is a residual stress measurement method generally used in isotropic materials. This study provides an evaluation into applying DHD to determine residual and fit-up stress fields in anisotropic composite materials. In this method, a reference hole with a small diameter is firstly drilled through the thickness of the component. The diameter of the hole is measured accurately and then a cylindrical core of material around the hole is trepanned from the component, relaxing the residual stresses in the core. Finally, the diameter of the reference hole is re-measured and the change in diameter used to calculate the residual stress. For anisotropic materials, the calculation of residual stresses requires the evaluation of distortion coefficients which rely on the mechanical properties of the components. In this work, Lekhnitskii'solution for the distortion of a circular hole in an anisotropic plate and finite element analysis are used to determine these coefficients. Using this technique, the in-plane residual stresses in AS4/8552 composite laminates were experimentally measured and compared to finite element predictions as well as to classical lamination theory. The obtained results indicate that, when using DHD in laminated materials, an optimal ratio between the layers' thickness, the reference hole and trepan diameters is of significant importance since unrelaxed thermally induced residual stresses can lead to inaccurate measurements.
\end{abstract}

\section{Introduction}

Deep-hole drilling (DHD) is a technique that allows the measurement of residual stress fields through very thick components. This technique requires that a small diameter hole is drilled through a component that contains residual stresses. The hole diameter is accurately measured (generally using an air probe) as a function of both depth and angular position inside the hole. The residual stresses in the component are afterwards released by machining away a core of larger diameter from around the hole. The relaxation of residual stress distorts the shape of the reference hole. The reference hole diameter is subsequently re-measured at the same angular positions and depths. The reference hole distortion is lastly related to the residual stresses that existed in the component before drilling the hole.

The main assumption of this method is that the drilling of the reference hole has no effect on the residual stress state and that removing the core causes the residual stresses around the hole to be fully released in an elastic manner [1]. The core is additionally assumed to consist of independent lengths. 
This indicates that a thick specimen is equivalent to a set of layers unconnected by through-thickness shear stresses [2]. The deep hole drilling technique has usually been used on metal parts such as in welds [3]-[5] and railway tracks [6]. In metallic components, EDM can be used to trepan a central core around the reference hole since the cutting stresses introduced by this method are not significant. An attempt to apply this technique to a laminated carbon-fibre composite was made by Bateman et al. [2]. In their investigation, EDM could not be used to machine the core around the reference hole because CFRP is not conductive and for this reason a diamond encrusted hole saw was selected instead, as it was for this study as well.

\section{Analytical model}

The DHD technique is based on the measured radial distortion to determine the components of the residual stress from the reference hole. The formulation to the case of far field biaxial stresses plus shear stress can be stated as

$$
\bar{u}=\frac{1}{E}\left[f_{\theta_{i}} \sigma_{x}+g_{\theta_{i}} \sigma_{y}+h_{\theta_{i}} \tau_{x y}\right]
$$

where for the case of isotropic materials (measurement angle $\theta$ )

$$
\begin{aligned}
& f_{\theta_{i}}=1+2 \cos 2 \theta_{i} \\
& g_{\theta_{i}}=1-2 \cos 2 \theta_{i} \\
& h_{\theta_{i}}=4 \sin 2 \theta_{i}
\end{aligned}
$$

In the case of orthotropic materials these distortion coefficients depend on the mechanical properties of the component. Additionally, the relationship between the longitudinal direction of the material (fibre direction) and the measurement angle (air probe angular position during experiments) must be taken into consideration, see Figure 1.

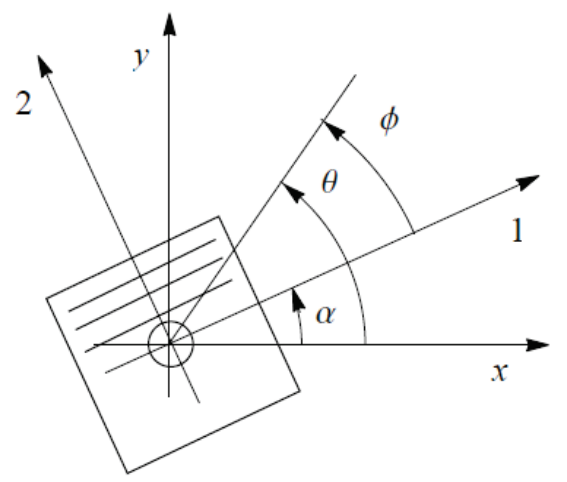

Fig. 1: Unidirectional laminate showing the relationship between the fibre direction ( $\alpha)$ and measurement $(\theta)$ angles in reference to the global axes.

For this analysis, these coefficients for the studied material (AS4/8852) were calculated using the finite element software ABAQUS considering the mechanical properties given in Table 1 and the following values were determined

$$
\begin{aligned}
f_{\theta_{i}} & =1.49+5.21 \cos 2 \phi_{i} \\
g_{\theta_{i}} & =15.89-19.51 \cos 2 \phi_{i} \\
h_{\theta_{i}} & =30.04 \sin 2 \phi_{i}
\end{aligned}
$$




\begin{tabular}{ccc}
\hline $\begin{array}{c}\text { Engineering } \\
\text { constant }\end{array}$ & & Value \\
\hline$E_{1}$ & {$[\mathrm{GPa}]$} & 135 \\
$E_{2}$ & {$[\mathrm{GPa}]$} & 9.6 \\
$E_{3}$ & {$[\mathrm{GPa}]$} & 9.6 \\
$\sigma_{12}$ & - & 0.3 \\
$\sigma_{13}$ & - & 0.3 \\
$\sigma_{23}$ & - & 0.435 \\
$G_{12}$ & {$[\mathrm{GPa}]$} & 5.2 \\
$G_{13}$ & {$[\mathrm{GPa}]$} & 5.2 \\
$G_{23}$ & {$[\mathrm{GPa}]$} & 3.4 \\
\hline
\end{tabular}

Table 1: Mechanical properties of AS4/8552.

The radial distortion is normally measured at nine different angles and at least three angles are required to obtain accurate results. The relation of the measured radial distortions and the residual stress is given by

$$
\overline{\mathbf{u}}=-\frac{1}{E} \mathbf{M} \cdot \boldsymbol{\sigma}
$$

The use of the minus sign is required because the radial distortions are measured after releasing the residual stresses. The matrices are expressed as

$$
\overline{\mathbf{u}}=\left[\begin{array}{c}
\left.\bar{u}\right|_{\phi=\phi_{1}} \\
\vdots \\
\left.\bar{u}\right|_{\phi=\phi_{i}} \\
\vdots \\
\left.\bar{u}\right|_{\phi=\phi_{N}}
\end{array}\right], \quad \mathbf{M}=\left[\begin{array}{ccc}
f_{\phi_{1}} & g_{\phi_{1}} & h_{\phi_{1}} \\
\vdots & \vdots & \vdots \\
f_{\phi_{i}} & g_{\phi_{i}} & h_{\phi_{i}} \\
\vdots & \vdots & \vdots \\
f_{\phi_{N}} & g_{\phi_{N}} & h_{\phi_{N}}
\end{array}\right] \quad \text { and } \quad \boldsymbol{\sigma}=\left[\begin{array}{c}
\sigma_{x} \\
\sigma_{y} \\
\tau_{x y}
\end{array}\right]
$$

where $\mathrm{N}$ is the number of angular measurements. Lastly, the residual stresses are determined from the measurements of the radial distortions of the hole by

$$
\boldsymbol{\sigma}=-E \mathbf{M}^{*} \cdot \overline{\mathbf{u}}
$$

where $\mathbf{M}^{*}=\left(\mathbf{M}^{\mathbf{T}} \cdot \mathbf{M}\right)^{-1} \cdot \mathbf{M}^{\mathbf{T}}$ is the pseudo-inverse of $\mathbf{M}$.

\section{Experimental procedures and results}

An AS4/8552 composite laminate of $18 \mathrm{~mm}$ in thickness consisting of 10 layers with different orientations was used to perform the DHD measurement, see Figure 2. To allow the measurement of residual stress using DHD, front and rear bushes were bonded using high strength adhesive to the specimen, centred on the location of the measurement.

The use of bushes has several benefits provide a setup for the fixation of the essential components for gun drilling and air probing and contribute to guide the hole saw perpendicularly to the specimen. Additionally, they decrease the effects of the air probe entrance and exit during the measurements of the diameters and help to avoid fibres delamination when the drill enters and exits the specimen. 


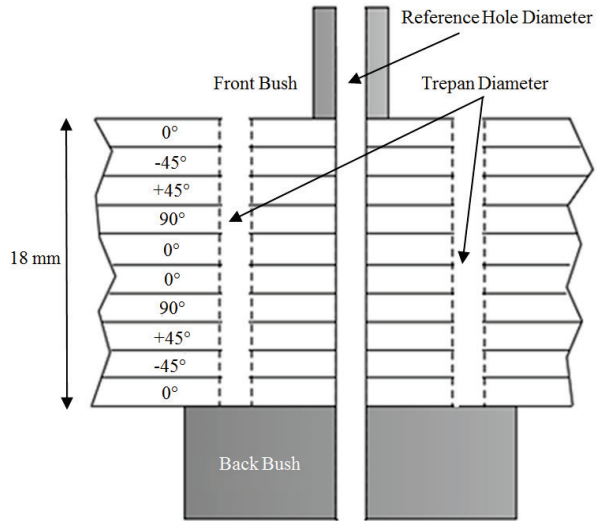

(a)

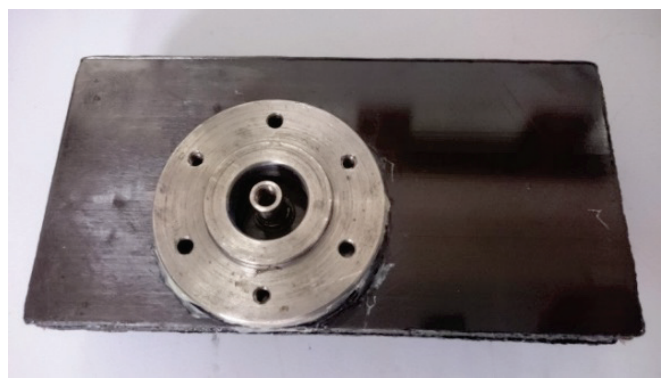

(b)

Fig. 2: (a) Illustration showing the layer arrangement specimens and the bushes required for a DHD experiment (b) composite laminate after conducting the DHD experiment

After drilling the reference hole, the air probe was introduced to measure precisely the hole diameter every $22.5^{\circ}$ (i.e. $0^{\circ}, 22.5^{\circ}, 45^{\circ}, 67.5^{\circ}, 90^{\circ}, 112.5^{\circ}, 135^{\circ}, 157.5^{\circ}$ and $180^{\circ}$ ) around it and at every $0.1 \mathrm{~mm}$ through it, resulting in nine measurements of diameter in total for every axial position. The air probe is not able measure accurately near a surface, nevertheless the front and rear bushes allow measurements near the surface of the specimens. The air probe must be calibrated before and after the measurements of the hole diameters using 10 calibration rings, which allow to use calibration curves and interpret a diameter to each reading in millivolts. The calibration rings used have the following dimensions: 2.9796, 2.9893, 2.9997, 3.0096, 3.0204, 3.0306, 3.0397, 3.0496, 3.0595 and $3.0704 \mathrm{~mm}$.

A hole saw was selected to perform the trepanning process which has the advantages of being faster than electrical discharge machining (EDM) and not requiring an electrolyte to be used. The hole saw used during the experiment has a diameter of $10 \mathrm{~mm}$ and is diamond tipped. Following the trepanning procedure, the diameter of the hole was re-measured using the air probe.

Lastly, the residual stresses were determined from the radial distortions of the reference hole to reveal the residual stress distributions shown in Figure 3. The principal material directions have been used in each layer. Therefore the stress component $\sigma_{11}$ is longitudinal to the fibre direction while the stress component $\sigma_{22}$ is in the transverse direction of the fibres. The experimental results are compared to Laminator [7] (software based on classical laminate theory) predictions and DHD simulations using the same reference hole and trepan diameters as in the experiments as well as different sizes of these two parameters additionally. The highest and lowest predicted stresses in the longitudinal direction are of the order of 20 and $65 \mathrm{MPa}$ in compression, in the $0^{\circ}$ and $90^{\circ}$ plies respectively. On the other hand, the expected stresses in the transverse direction to the fibres remained approximately on 36-38 $\mathrm{MPa}$ on the specimen. The predicted magnitude of the shear stress is very low throughout the entire laminate.

A comparison between the DHD experiment and the DHD simulation using a reference hole of 3 $\mathrm{mm}$ of diameter and a trepan diameter of $10 \mathrm{~mm}$ shows a close agreement in the longitudinal, transversal direction as well as the in-plane shear component. However, these values significantly disagree with residual stress predictions using Laminator, which implies that a considerable amount of residual stresses were not relaxed after trepanning the core. For the proceeding of the DHD analysis, the core thickness is assumed to consist of a number of independent sections, each confined by two parallel planes normal to the reference hole axis. Each section is totally unaffected by the presence of other sections. As a consequence of the trepanning process, the stresses inside the core are totally released in a linear-elastic way. 
(a)

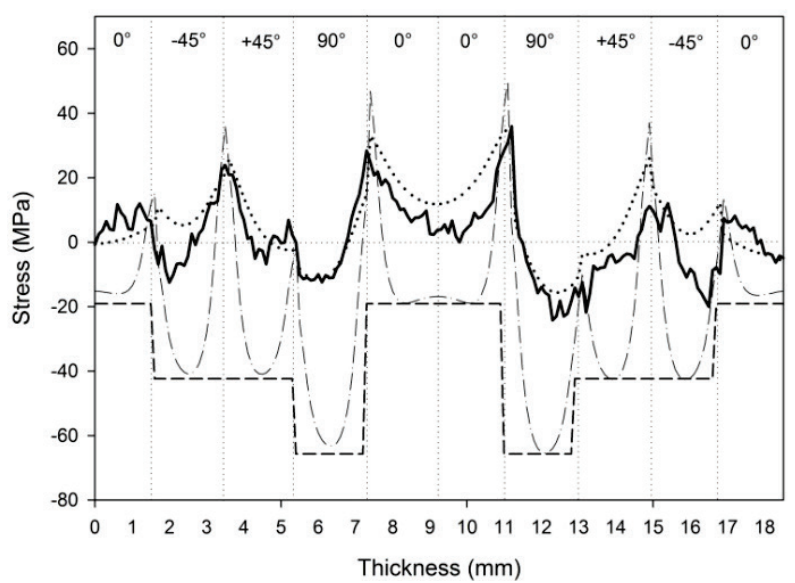

(b)

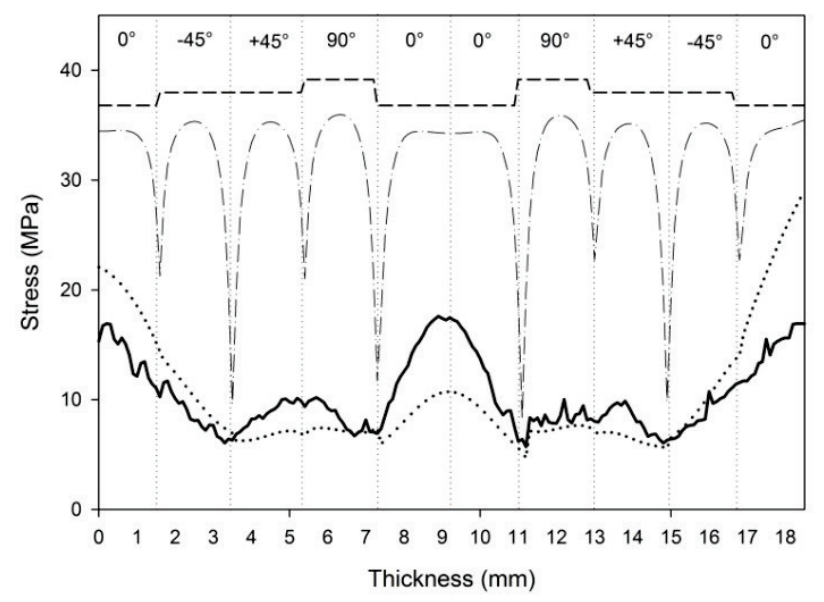

(c)
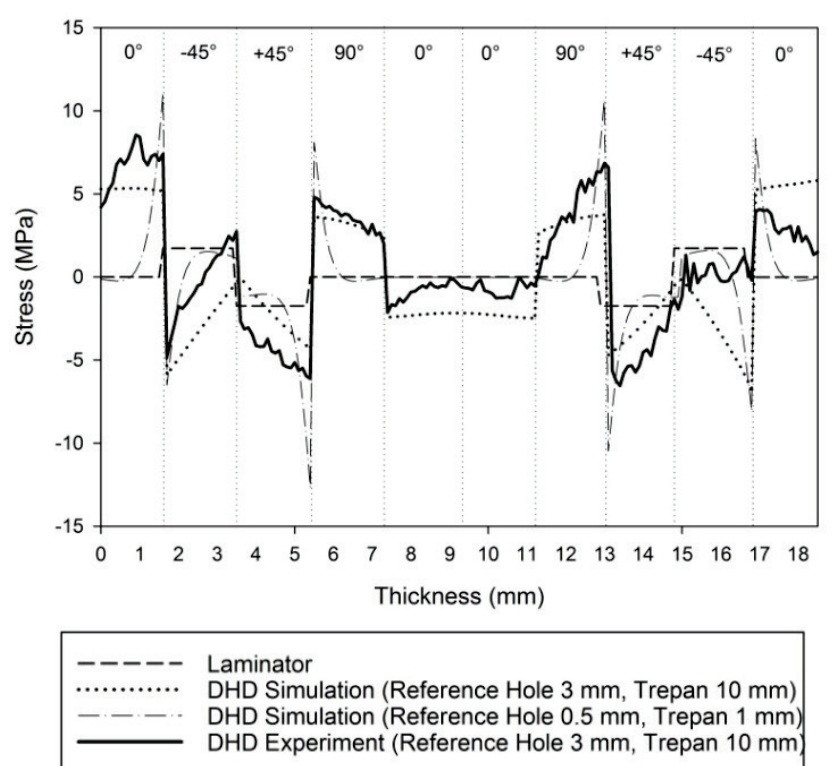

Fig. 3: Comparison of the residual stresses $\sigma_{11}(a), \sigma_{22}(b)$ and in-plane shear $\tau_{12}(c)$ using Laminator, DHD experiment and simulations with different reference hole and trepan diameters.

In the case of composite laminates, these block lengths (layers) are influenced by the presence of adjacent layers since their stiffness and coefficient of thermal expansion variation may be sometimes higher than 15 times, thus affecting the area near their interface when expanding and contracting during the cure process. For this reason, the assumption that the trepanning process relaxes all 
residual stresses in the core cannot be valid. This issue can be nevertheless considerably resolved by using a smaller dimension for the reference hole and trepan diameters. After selecting $0.5 \mathrm{~mm}$ and 1 $\mathrm{mm}$ for the reference hole and trepan diameters respectively in a further DHD simulation the disagreement between the obtained results and Laminator predictions did not exceed a value of 5 $\mathrm{MPa}$ in all three cases in the central area of each layer.

\section{Summary}

The deep-hole drilling technique has become a standard method for the measurement of residual stress in isotropic materials, especially for thick components. The work described here is an extension to the method to allow the measurement of residual stress in orthotropic materials such as thick composite laminates.

After conducting a deep-hole drilling experiment on an $18 \mathrm{~mm}$ thick AS4/8552 composite plate, measurements showed a good agreement with a simulation using the same reference hole and trepan parameters, but a significant discrepancy was found when compared with correct predictions (Laminator). It was determined that, when using DHD in laminated materials, the reference hole and trepan diameters must be much smaller than the thickness of the layers in order to reduce the effect of remaining interlaminar shear stresses in trepanned cores which can significantly lead to inaccurate measurements.

\section{Acknowledgements}

The authors would like to acknowledge Mr. Steve Harding and VEQTER Ltd. for providing technical support for the experimental studies. The travel grant awarded by the Alumni Foundation of the University of Bristol as well as the financial support for this project provided by the National Council for Science and Technology (CONACyT), the Institute of Innovation and Technology Transfer $\left(\mathrm{I}^{2} \mathrm{~T}^{2}\right)$ and the Government of Nuevo León are likewise gratefully acknowledged.

\section{References}

[1] A. Mirzaee-Sisan, A. J. Fookes, C. E. Truman, D. J. Smith, T. B. Brown, and T. A. Dauda, "Residual stress measurement in a repair welded header in the as-welded condition and after advanced post weld treatment," International Journal of Pressure Vessels and Piping, vol. 84, no. 5, pp. 265-273, 2007. http://dx.doi.org/10.1016/j.ijpvp.2007.01.003

[2] M. G. Bateman, O. H. Miller, T. J. Palmer, C. E. P. Breen, E. J. Kingston, D. J. Smith, and M. J. Pavier, "Measurement of residual stress in thick section composite laminates using the deep-hole method," International Journal of Mechanical Sciences, vol. 47, no. 11, pp. 17181739, 2005. http://dx.doi.org/10.1016/j.ijmecsci.2005.06.011

[3] P. J. Bouchard, D. George, J. R. Santisteban, G. Bruno, M. Dutta, L. Edwards, E. Kingston, and D. J. Smith, "Measurement of the residual stresses in a stainless steel pipe girth weld containing long and short repairs," International Journal of Pressure Vessels and Piping, vol. 82, no. 4, pp. 299-310, Apr. 2005. http://dx.doi.org/10.1016/j.ijpvp.2004.08.008

[4] T. B. Brown, T. A. Dauda, C. E. Truman, D. J. Smith, D. Memhard, and W. Pfeiffer, "Predictions and measurements of residual stress in repair welds in plates," International Journal of Pressure Vessels and Piping, vol. 83, no. 11-12, pp. 809-818, 2006. http://dx.doi.org/10.1016/j.ijpvp.2006.08.012

[5] D. George and D. J. Smith, "Through thickness measurement of residual stresses in a stainless steel cylinder containing shallow and deep weld repairs," International Journal of Pressure Vessels and Piping, vol. 82, no. 4, pp. 279-287, 2005.

http://dx.doi.org/10.1016/j.ijpvp.2004.08.006

[6] D. Stefanescu, P. A. Browne, C. E. Truman, and D. J. Smith, "Residual Stress Measurement within a European UIC60 Rail Using Integrated Drilling Techniques," Materials Science Forum, vol. 440-441, pp. 85-92, 2003. http://dx.doi.org/10.4028/www.scientific.net/MSF.440-441.85

[7] M. Lindell, "The Laminator: Classical Analysis of Composite Laminates (Version 3.7). 